(2) Open Access Full Text Article

\title{
Intracameral ketorolac concentration at the beginning and end of cataract surgery following preoperative topical ketorolac administration
}

This article was published in the following Dove Press journal:

Clinical Ophthalmology

24 October 2017

Number of times this article has been viewed

\author{
Douglas A Katsev' \\ Cailyn C Katsev' \\ Jen Pinnow ${ }^{2}$ \\ Catherine $M$ Lockhart $^{3}$ \\ 'Santa Barbara Surgery Center, \\ Santa Barbara, CA, ${ }^{2}$ Pinnow \\ Consulting, Bellingham, WA, ${ }^{3}$ Omeros \\ Corporation, Seattle, WA, USA
}

Purpose: To evaluate the intracameral concentration of ketorolac tromethamine (ketorolac) at the beginning and end of cataract surgery following preoperative topical administration.

Setting: Santa Barbara Surgery Center, Santa Barbara, CA, USA.

Design: This was a pre-post, interventional, single-arm study.

Patients and methods: Patients undergoing cataract extraction and lens replacement (CELR) were eligible. Written informed consent was obtained from 14 patients who were prescribed topical ophthalmic ketorolac according to the surgeon's usual practice beginning the day prior to surgery. The surgeon withdrew $100 \mu \mathrm{L}$ of aqueous humor from the operative eye immediately prior to the initial surgical incision and again just prior to final anterior chamber reinflation and wound closure. Ketorolac concentrations in the intracameral fluid samples were measured.

Results: Thirteen of 14 patients used four doses of ketorolac the day prior to surgery as directed, and one patient used three doses. On the day of surgery, all 14 patients administered one drop of topical ketorolac on awakening and one drop after arriving at the surgery center. Preoperative ketorolac concentrations for the 12 patients from whom samples were collected ranged from 4.9 to $369 \mathrm{ng} / \mathrm{mL}$. End-of-procedure sample concentrations ranged from $<1.0$ (the lower limit of quantification [LLOQ]) to $6.32 \mathrm{ng} / \mathrm{mL}$, with eight of 12 patients having ketorolac levels below the LLOQ.

Conclusion: At-home compliance with topical ketorolac was good, with $92.9 \%$ of patients using it as directed. Following CELR, levels of ketorolac in the aqueous humor were low, and $66.7 \%$ of patients had undetectable levels.

Keywords: cataract surgery, NSAIDs, ketorolac, topical administration, intracameral drug concentration

\section{Introduction}

Age-related cataract is the leading cause of vision impairment in the United States and worldwide, representing approximately one-third of the vision-impaired population. ${ }^{1-3}$ Cataracts are usually asymptomatic but, as a progressive disease, once vision is affected there is a steady and irreversible decline in visual function. ${ }^{4}$ When cataracts progress enough to impair visual function, they become clinically relevant and the primary treatment is surgery. ${ }^{4,5}$

Many surgeons prescribe topical medications to be used pre- and postoperatively for infection prophylaxis and to prevent inflammation and reduce pain. ${ }^{4,6}$ Ocular inflammation is common after cataract surgery. Untreated inflammation can cause pain and other complications such as increased intraocular pressure and cystoid macular edema (CME). In an attempt to prevent or reduce inflammation and therefore reduce
Correspondence: Catherine M Lockhart Omeros Corporation, 20I Elliott Ave W, Seattle, WA 98119 USA

Email clockhart@omeros.com 
surgical pain and the risk of CME, surgeons commonly prescribe preoperative nonsteroidal anti-inflammatory drugs (NSAIDs) to patients undergoing cataract surgery. ${ }^{6,7}$

Physiologically, NSAIDs inhibit cyclooxygenase enzymes (COX-1 and COX-2), which are responsible for initiating the inflammatory cascade through prostaglandin synthesis. Prostaglandin release, a known result of surgical trauma, is associated with pain and inflammation and also with stimulation and contraction of the iris sphincter muscle, resulting in pupil constriction. By inhibiting $\mathrm{COX}-1$ and $\mathrm{COX}-2$, NSAIDs prevent miosis caused by prostaglandins released during surgical insult and inhibit the inflammatory cascade that leads to pain and other sequelae such as CME. ${ }^{7}$

While NSAIDs are known to inhibit mechanistically the inflammatory cascade, there are potential limitations associated with topical administration of NSAIDs as a way to control inflammation during and after cataract surgery. First, preoperative topical NSAID use often requires multiple, selfadministered doses over several days to allow for adequate permeation into ocular tissues, thus patient adherence with the regimen is important. ${ }^{8}$ If doses are missed, the therapeutic effect may be diminished. Second, while there may be some depot effect in ocular tissues with proper dosing and administration, any active drug present at the start of surgery may be washed out of the aqueous humor by the standard irrigation method utilized during surgery. There are no known studies that examine the extent to which preoperative topical medications used in ocular surgery may be washed out by surgical irrigation.

The purpose of this study was to evaluate the intracameral concentration of ketorolac tromethamine (ketorolac) at the beginning and end of cataract surgery following preoperative topical administration of ketorolac.

\section{Patients and methods}

This study was conducted at a single site and all surgical procedures were performed by a single surgeon. Patients undergoing unilateral cataract surgery and receiving preoperative ketorolac were eligible to participate. All patients provided written, informed consent to participate in this study. This study was approved by a central Institutional Review Board (Quorum, Seattle, WA, USA). Patients were excluded if they were using ophthalmic or systemic ketorolac at the time of screening, had extra- or intraocular inflammation present in either eye at the time of screening, had participated in any investigational drug or device study within 30 days prior to the day of surgery, or had any condition that the investigator deemed would put the patient at risk of harm or would confound the interpretation of the study data.

Patients received topical ketorolac for self-administration four times per day beginning 1 day prior to cataract surgery, according to the usual practice of the surgeon. Topical ketorolac was also administered upon awakening on the day of surgery and once in the surgical center according to the surgeon's normal practice.

On the day of surgery, patients were asked about their ketorolac use on the day prior to and on the morning of surgery to evaluate adherence. Immediately prior to the initial surgical incision, the surgeon withdrew a $100 \mu \mathrm{L}$ sample of aqueous humor from the operative eye with a 30-gauge needle attached to a tuberculin syringe. The fluid was withdrawn through the site of the corneal incision used to perform the surgical procedure. Irrigation solution was used liberally and as deemed necessary according to usual practice of the surgeon. At the end of the surgical procedure prior to final anterior chamber reinflation and wound closure, the surgeon withdrew another $100 \mu \mathrm{L}$ sample of fluid from the anterior chamber, which in addition to aqueous humor included surgical irrigation fluid and any fluids released from the anterior chamber structures.

The intracameral fluid samples were labeled and transported to an analytical laboratory (Alturas Analytics, Inc., Moscow, ID, USA) for analysis of ketorolac concentration at the time of surgical incision and at the end of the surgical procedure.

\section{Statistical analysis}

Given the anticipated variability across patients, results of ketorolac concentrations at the start and end of surgery are presented by individual patient rather than simply presenting the mean across all patients. A nonparametric Wilcoxon matched-pairs signed-rank test was used to compare ketorolac measurements before and after surgery, with imputation of a value of 1.0 for each measurement that was recorded as undetectable. The key assumption for this test is that the distribution of the data is the same for measurements at the beginning and end of surgery, but the distribution may not necessarily be normal. Statistical analysis was performed using Stata v 11.0 (StataCorp LP, College Station, TX, USA).

\section{Results}

A total of 14 patients met the inclusion criteria and were enrolled in this study. Table 1 describes the overall patient demographics. All patients received topical ketorolac for 
Table I Demographic information for enrolled patients

\begin{tabular}{ll}
\hline Patient characteristics & No of patients \\
\hline Total enrolled, $\mathrm{n}$ & 14 \\
Male, $\mathrm{n}(\%)$ & $9(64)$ \\
Age, mean years (range) & $68(52-84)$ \\
Race & \\
$\quad$ White & 13 \\
$\quad$ African American & $\mathrm{I}$ \\
Ethnicity & \\
Hispanic or Latino & 3 \\
Non-Hispanic or Non-Latino & 10 \\
Unknown & $\mathrm{I}$ \\
\hline
\end{tabular}

self-administration of four doses on the day prior to surgery. Thirteen patients $(92.9 \%)$ used all four doses, while one patient $(7.14 \%)$ only used three doses on the day prior to surgery. All patients also received two doses of topical ketorolac on the day of surgery, one at home and one after arrival at the surgical center. Aqueous humor samples were inadvertently not collected from two patients, leaving a total of 12 patients for full analysis.

Ketorolac concentrations were analyzed from the fluid samples drawn from the anterior chamber immediately prior to the first surgical incision and at the end of the surgical procedure in each patient. Preoperative ketorolac concentrations for the 12 patients with complete data ranged from 4.9 to $369 \mathrm{ng} / \mathrm{mL}$ (Table 2). At the end of the procedure, ketorolac concentrations ranged from undetectable (ie, below the lower limit of quantitation [LLOQ; $<1.00 \mathrm{ng} / \mathrm{mL}$ ]) to $6.32 \mathrm{ng} / \mathrm{mL}$. A total of eight patients (66.7\%) had ketorolac concentrations that were undetectable. The concentrations at the end of the procedure in the four patients with detectable concentrations of ketorolac were very low and clinically

Table 2 Ketorolac concentration in the aqueous humor sampled just prior to surgery and at the end of the surgical procedure

\begin{tabular}{lll}
\hline Patient & \multicolumn{2}{l}{ Ketorolac concentration $(\mathbf{n g} / \mathbf{m L})$} \\
\cline { 2 - 3 } number & Prior to incision & End of procedure \\
\hline 001 & 87.4 & Undetectable* \\
003 & 39.0 & Undetectable \\
004 & 4.90 & Undetectable \\
006 & 195 & Undetectable \\
007 & 29.5 & 1.8 \\
008 & 75.1 & 6.32 \\
009 & 369 & 2.11 \\
010 & 105 & Undetectable \\
011 & 244 & 2.90 \\
012 & 64.6 & Undetectable \\
013 & 137 & Undetectable \\
014 & 120 & Undetectable \\
\hline
\end{tabular}

Notes: $*$ Undetectable $=$ below lower limit of quantification (ie, $<1.00 \mathrm{ng} / \mathrm{mL}$ ); $p=0.0022$ (Wilcoxon signed-rank test). irrelevant, ranging from 1.8 to $6.32 \mathrm{ng} / \mathrm{mL}$. The difference in measured concentrations before and after surgery was significant $(p=0.0022)$.

\section{Discussion}

This was a single-site, single-arm study to assess the concentration of ketorolac present in the aqueous humor at the start and end of routine cataract surgery in patients who were treated with topical ketorolac prior to surgery. A statistically significant difference was shown between pre- and postsurgical concentrations of ketorolac measured in the fluid drawn from the anterior chamber $(p=0.0022)$. A post hoc power calculation based on the means and standard deviations calculated pre- and post-surgery for the overall group showed $95.7 \%$ power based on the sample size in this study.

It is common practice to administer topical NSAIDs (eg, ketorolac) for at least 1 day prior to cataract surgery in order to reduce inflammation induced by surgical insult and, therefore, to reduce pain and other complications associated with an inflammatory reaction inside the eye. As the eye is a physiologically privileged organ system, it takes multiple topical doses of ketorolac to adequately penetrate the anterior chamber and reach the ocular tissues that are affected during cataract surgery. This requires good patient adherence to the dosing regimen. During cataract surgery, the cornea is penetrated and the anterior chamber is bathed with standard ophthalmic irrigation solution to maintain fluid balance and structural integrity of the eye. It is a reasonable possibility that any residual ketorolac present in the aqueous humor due to topical administration would be washed out during surgery with the standard irrigation, reducing or eliminating the intended anti-miotic and anti-inflammatory effects so important during and after surgery. The extent to which standard irrigation affects localized ketorolac concentrations at the site of the target tissues in the anterior chamber following preoperative topical administration has not previously been investigated.

In this study, all patients received topical ketorolac on the day prior to surgery, and at-home adherence to the dosing regimen was good, with $92.9 \%$ of patients using the drug as directed, and only one patient missing just one of the prescribed four doses on that day. All patients received two doses of topical ketorolac on the day of surgery. Surgical procedures were performed by the same surgeon at a single clinic, so there was no systematic variability in patient care based on surgeon preference, facility process, or surgeon experience. All patients had detectable intracameral concentrations of ketorolac at the start of surgery. Although patient 
compliance was high and there were measurable amounts of ketorolac present in the aqueous humor at the start of surgery for all patients, the majority $(66.7 \%)$ of patients had undetectable amounts of ketorolac in the anterior chamber by the end of surgery with the remainder having very low and clinically irrelevant levels of measured ketorolac postoperatively. It is impossible to quantify the true inter-individual variability in these ketorolac measurements, as most were below the LLOQ of the assay instrumentation. It is not surprising to observe some inter-individual differences in these measurements, as the surgical conditions reflected real-world practice in every respect except for withdrawal of small fluid aliquots from the anterior chamber before and after surgery.

Studies have shown that treatment with NSAIDs reduces surgically induced miosis by inhibiting prostaglandin synthesis in anterior chamber tissues, although miosis often does occur. ${ }^{9-11}$ Similarly, by preventing prostaglandin synthesis, topical NSAIDs have been shown to reduce ocular inflammation after cataract surgery. ${ }^{10}$

CME is a complication related to cataract surgery that is the leading cause of post-surgical vision loss. ${ }^{10,12}$ Believed to be caused, in part, by an inflammatory response, many patients are treated with a prophylactic regimen of NSAIDs after cataract surgery. ${ }^{10}$ It remains unclear, however, whether postoperative administration of NSAIDs prevents CME or decreases the severity of disease, as the inflammatory response has already been initiated intraoperatively by surgical trauma.

In a study conducted in a real-world surgical setting such as this, it is impractical and inappropriate to measure tissue concentrations of drug as that would require biopsy or other tissue sample. This study was conducted to evaluate whether or not the current standard practice patterns of instilling a topical NSAID prior to surgery is likely to continue a therapeutic effect during or after surgery is completed. The clinical implications following washout of topical NSAIDs are not clear, as this does not account for any depot effect that may occur; however, in removing any free drug present in the eye, there is unlikely to be any continued effect as any remaining tissue-bound drug is physiologically cleared. Pharmacokinetic studies have shown the intraocular half-life of ketorolac to be 2.3 hours $^{13}$ and the half-maximal inhibitory concentration $\left(\mathrm{IC}_{50}\right)$ of COX-1 and $\mathrm{COX}-2$ by ketorolac to be $2 \times 10^{-8} \mathrm{M}$ and $12 \times 10^{-8} \mathrm{M},{ }^{14}$ respectively, so reducing aqueous humor levels to below the LLOQ is likely to remove the therapeutic effect.

Even though patient compliance with the preoperative ketorolac regimen was good in this study, the absence of ketorolac in the anterior chamber at the end of surgery suggests that preoperative topical delivery of ketorolac may not have the desired effect during and after the surgical procedure, times when the effects of an NSAID are needed the most. Concentrations of ketorolac in the anterior chamber immediately after surgery are important because tissues such as the iris/ciliary body are in contact with the fluid in the anterior chamber and are known sources of prostaglandin production. In order to achieve the desired intra- and postoperative anti-inflammatory effect, intracameral delivery of ketorolac or a ketorolac-containing drug during surgery may be necessary, providing preemptive inhibition of surgically induced prostaglandin release rather than trying to "undo" the intraoperative initiation of the inflammatory cascade with postoperative NSAID topical drops.

This study is limited by small sample size, but even with only 12 patients completing the study, the effect size is sufficiently large to be convincing. All patients had ketorolac levels that were easily detectable prior to surgery and had levels that were very low or below the LLOQ by the end of surgery.

In this study, the concentrations of ketorolac present in the anterior chamber of the eye both before and immediately after cataract surgery were quantified. The results showed that drug concentrations present after topical administration of ketorolac are dramatically reduced due to washout by the irrigation solutions used during the surgical procedure. This suggests that the anticipated therapeutic benefit of topical ketorolac may not persist during and after surgery.

\section{Acknowledgments}

This study was funded by Omeros Corporation, Seattle, WA, USA. This work was presented as a poster at the American Society of Cataract and Refractive Surgery - American Society of Ophthalmic Administrators Symposium \& Congress, San Diego, CA, USA, April 17-21, 2015.

\section{Disclosure}

DA Katsev and J Pinnow are consultants for Omeros Corporation and J Pinnow holds an equity interest in Omeros. CM Lockhart is an employee of Omeros Corporation. CC Katsev has no financial interest in the subject matter of this manuscript. The authors report no other conflicts of interest in this work.

\section{References}

1. Bourne RR, Jonas JB, Flaxman SR, et al. Prevalence and causes of vision loss in high-income countries and in Eastern and Central Europe: 1990-2010. Br J Ophthalmol. 2014;98:629-638. 
2. Congdon N, Vingerling JR, Klein BE, et al. Prevalence of cataract and pseudophakia/aphakia among adults in the United States. Arch Ophthalmol. 2004;122:487-494.

3. Thylefors B. The World Health Organization's programme for the prevention of blindness. Int Ophthalmol. 1990;14:211-219.

4. Preferred Practice Pattern ${ }^{\circledR}$ Guidelines. Cataract in the adult eye. American Academy of Ophthalmology; 2011. Available from: http:// www.aao.org/ppp. Accessed September, 2016.

5. Asbell PA, Dualan I, Mindel J, Brocks D, Ahmad M, Epstein S. Agerelated cataract. Lancet. 2005;365:599-609.

6. Grob SR, Gonzalez-Gonzalez LA, Daly MK. Management of mydriasis and pain in cataract and intraocular lens surgery: review of current medications and future directions. Clin Ophthalmol. 2014;8:1281-1289.

7. O'Brien TP. Emerging guidelines for use of NSAID therapy to optimize cataract surgery patient care. Curr Med Res Opin. 2005;21:1131-1137.

8. Donnenfeld ED. Dose-response curve of a topical NSAID as a surgical tool before cataract surgery. American Society of Cataract and Refractive Surgery - American Society of Ophthalmic Administrators (ASCRSASOA) Symposium \& Congress; April 16-20; 2005; Washington, DC.
9. Drews RC, Katsev DA. Ocufen and pupillary dilation during cataract surgery. J Cataract Refract Surg. 1989;15:445-448.

10. Kim SJ, Flach AJ, Jampol LM. Nonsteroidal anti-inflammatory drugs in ophthalmology. Surv Ophthalmol. 2010;55:108-133.

11. Srinivasan R, Madhavaranga. Topical ketorolac tromethamine $0.5 \%$ versus diclofenac sodium $0.1 \%$ to inhibit miosis during cataract surgery. J Cataract Refract Surg. 2002;28:517-520.

12. Colin J. The role of NSAIDs in the management of postoperative ophthalmic inflammation. Drugs. 2007;67:1291-1308.

13. Margalit E, Kugler LJ, Brumm MV, et al. The safety of intraocular ketorolac in rabbits. Invest Ophthalmol Vis Sci. 2006;47:2093-2099.

14. Waterbury LD, Galindo D, Villanueva L, et al. Ocular penetration and anti-inflammatory activity of ketorolac $0.45 \%$ and bromfenac $0.09 \%$ against lipopolysaccharide-induced inflammation. J Ocul Pharmacol Ther. 2011;27:173-178.
Clinical Ophthalmology

\section{Publish your work in this journal}

Clinical Ophthalmology is an international, peer-reviewed journa covering all subspecialties within ophthalmology. Key topics include: Optometry; Visual science; Pharmacology and drug therapy in eye diseases; Basic Sciences; Primary and Secondary eye care; Patient Safety and Quality of Care Improvements. This journal is indexed on

Submit your manuscript here: http://www.dovepress.com/clinical-ophthalmology-journal

\section{Dovepress}

PubMed Central and CAS, and is the official journal of The Society of Clinical Ophthalmology (SCO). The manuscript management system is completely online and includes a very quick and fair peer-review system, which is all easy to use. Visit http://www.dovepress.com/ testimonials.php to read real quotes from published authors. 